Int. J. Morphol.,

32(1):32-35, 2014.

\title{
Evaluación de la Posición Condilar y del Espacio Articular en ATM de Individuos Chilenos con Trastornos Temporomandibulares
}

\author{
Assessment of TMJ Condylar Position and Joint Space in \\ Chilean Individuals with Temporomandibular Disorders
}

Alves, N." ; Deana, N. F.** ; Schilling, Q. A. ${ }^{* * *}$; González, V. A.****; Schilling, L. J.*** \& Pastenes, R. C.****

ALVES, N.; DEANA, N. F.; SCHILlING, Q. A.; GONZÁLEZ, V. A.; SCHILLING, L. J. \& PASTENES, R. C. Evaluación de la posición condilar y del espacio articular en ATM de individuos chilenos con trastornos temporomandibulares. Int. J. Morphol., 32(1):32$35,2014$.

RESUMEN: La importancia de la relación cóndilo-fosa en la articulación temporomandibular (ATM) aún no es clara, sin embargo algunos autores asocian la posición no céntrica del cóndilo de la mandíbula en la fosa mandibular con trastorno temporomandibular (TTM). Además, otros autores sugieren que la evaluación del espacio articular es una herramienta diagnóstica para la evaluación de las alteraciones de ATM. El objetivo de este estudio fue evaluar la posición condilar y el espacio articular de ATM en individuos chilenos con TTM utilizando tomografía computarizada cone-beam. Fueron seleccionados 19 pacientes de la Unidad de Trastornos Cráneo Cervico Mandibulares-Universidad de Talca, con diagnóstico de TTM según el Research Diagnostic Criteria for Temporomandibular Disorders. Para la evaluación de la posición condilar se utilizó la técnica de Pullinger \& Hollender y para la evaluación de los espacios articulares anterior (EAA), superior (EAS) y posterior (EAP) se utilizó la técnica de Ikeda \& Kawamura. Un 39,47\% de los cóndilos se presentaron en posición posterior, $34,21 \%$ en posición central y un 26,31\% en posición anterior. Para cóndilos en posición central encontramos: $\mathrm{EAA}=1,57 \mathrm{~mm} ; \mathrm{EAS}=2,20 \mathrm{~mm} ; \mathrm{EAP}=1,54 \mathrm{~mm}$. Para cóndilos en posición posterior encontramos: EAA=2,18 mm; EAS=2,02 mm; $\mathrm{EAP}=0,98 \mathrm{~mm}$. Para cóndilos en posición anterior encontramos: $\mathrm{EAA}=1,00 \mathrm{~mm}$; EAS $=1,89 \mathrm{~mm}$ y EAP=2,07 mm. Concluimos que individuos con diagnóstico de TTM presentan las regiones anatómicas predisponientes a las alteraciones articulares con espacios articulares disminuidos cuando comparados con sujetos asintomáticos. La posición posterior fue la más común en individuos con TTM, sin embargo hubo gran variabilidad en la posición condilar, por ello sugerimos que una posición no centralizada del cóndilo no está necesariamente asociada con la presencia de TTM. Concluimos además que en individuos con TTM los cóndilos ubicados en posición central y posterior presentan EAP disminuido en comparación con individuos sanos.

PALABRAS CLAVE: Posición condilar; Espacio articular; Articulación temporomandibular; Trastorno temporomandibular.

\section{INTRODUCCIÓN}

Los trastornos temporomandibulares (TTM) abarcan una amplia serie de desordenes funcionales en los cuales están implicados no sólo la articulación temporomandibular (ATM), sino también los músculos involucrados en los movimientos mandibulares, la musculatura cervical y estructuras asociadas (Alves \& Cândido, 2013).

La relación existente entre el cóndilo de la mandíbula y la fosa mandibular en la ATM ha sido objeto de varios estudios tanto en pacientes con síntomas como en pacientes sin síntomas de TTM y los resultados reportados en las investigaciones son antagónicos. Para Pullinger \& Hollender (1986) los individuos asintomáticos presentan cóndilos en posición central, corroborando con los resultados reportados por Pereira \& Gavião (2004) que indican que pacientes con TTM presentan cóndilos en posición posterior, o sea, no central. Ya Pullinger et al. (1985), asocian la relación cóndilo-fosa no central a la función normal de la ATM, ratificando los hallazgos de Katzberg et al. (1983), que no encontraron diferencias en la posición condilar de pacientes

* Departamento de Odontología Integral Adulto. Centro de Investigación CIMA, Facultad de Odontología, Universidad de La Frontera, Temuco, Chile.

** Kinesiólogo, Temuco, Chile.

**** Departamento de Estomatología, Facultad de Ciencias de la Salud, Universidad de Talca, Talca, Chile.

${ }^{* * * *}$ Departamento de Rehabilitación Buco-Maxilofacial, Facultad de Ciencias de la Salud, Universidad de Talca, Talca, Chile.

Este Proyecto fue Subvencionado por la Universidad de Talca, Resolución 444/2011. 
con graves trastornos internos de la ATM y de sujetos asintomáticos.

Kinniburgh et al. (2000), afirman que la medición del espacio articular proporciona una importante información para el diagnóstico de enfermedades de la ATM. Ellos observaron que mujeres con posición anterior del disco tenían espacios articulares superior y posterior reducidos y aumento del espacio articular anterior. Los varones con posición anterior del disco mostraron reducción del espacio articular superior, aumento del espacio articular anterior y posición condilar relativamente posterior. Sin embargo, otros estudios reportan que no existen diferencias en los espacios articulares (anterior, posterior y superior) entre individuos asintomáticos y sintomáticos (Matos et al., 2005).

El rol de la posición condilar y del espacio articular de la ATM en la etiología de los TTM todavía permanece un tema controvertido en la literatura. El objetivo de este estudio fue evaluar la posición condilar y el espacio articular de la ATM en individuos chilenos con diagnóstico clínico de TTM utilizando tomografía computarizada cone-beam (TCCB).

\section{MATERIAL Y MÉTODO}

Fueron seleccionados 19 pacientes chilenos, de ambos sexos, con edad entre 23 y 71 años (promedio de 48,5 años), asistidos en la Unidad de Trastornos Cráneo Cérvico Mandibulares (UCRACEM) - Universidad de Talca, Chile, que presentaban diagnóstico de TTM, grupo III, según el Research Diagnostic Criteria for Temporomandibular Disorders (RDC/TMD) (Dworkin \& Le Resche, 1992).

Para evaluación de la posición condilar se utilizó la ecuación: $\mathrm{DC}=(\mathrm{P}-\mathrm{A} / \mathrm{P}+\mathrm{A}) \times 100 \%$

Donde $\mathrm{DC}=$ desplazamiento condilar, $\mathrm{P}=$ espacio articular lineal posterior y $\mathrm{A}=$ espacio articular lineal anterior. Valor de $\mathrm{DC}=0$ representa una posición condilar céntrica absoluta; valores entre $-12 \%$ y $+12 \%=$ posición condilar céntrica; valores menores que $-12 \%=$ posición condilar posterior; valores mayores que $+12 \%=$ posición condilar anterior. Este método, descrito por Pullinger \& Hollender ha sido modificado por Pereira et al. (2007).

Las ATMs fueron separadas en 3 grupos de acuerdo con la posición condilar: grupo 1- central; grupo 2- posterior; grupo 3- anterior. Luego se analizaron los valores obtenidos para los espacios articulares (anterior, posterior y superior), en cada grupo.
Para la evaluación de los espacios articulares se utilizó el método descrito por Ikeda \& Kawamura (2009). Para ello, se realizó medidas lineales en reconstrucciones de imágenes sagitales obtenidas por tomografía computarizada conebeam (TCCB). En el punto más superior de la fosa mandibular fue trazada una línea horizontal utilizada como un plano de referencia y desde el mismo punto fueron trazadas líneas tangentes a los puntos anterior y posterior más prominentes de la cabeza de la mandíbula. Las distancias desde los puntos tangentes anterior y posterior hasta la fosa mandibular corresponden a los espacios articulares anterior (EAA) y posterior (EAP), respectivamente. La distancia desde el punto más superior de la cabeza de la mandíbula hasta el punto más superior de la fosa mandibular sobre la línea horizontal, corresponde al espacio articular superior (EAS) (Fig. 1).

Los valores obtenidos fueron analizados a través de la prueba Mann-Whitney. Se consideró estadísticamente significativo $\mathrm{p}<0,05$.

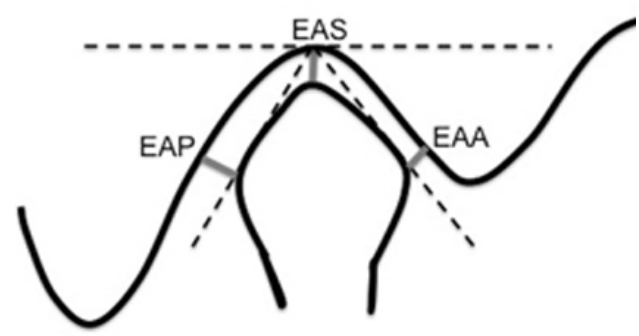

Fig. 1. EAS (espacio articular superior), EAP (espacio articular posterior), EAA (espacio articular anterior)

\section{RESULTADOS}

A través de la técnica de Pullinger \& Hollender encontramos un $39,48 \%$ de cóndilos en posición posterior (Fig. 2A), un 34,21\% de cóndilos en posición central (Fig. 2B) y un 26,31\% de cóndilos en posición anterior (Fig. 2C).

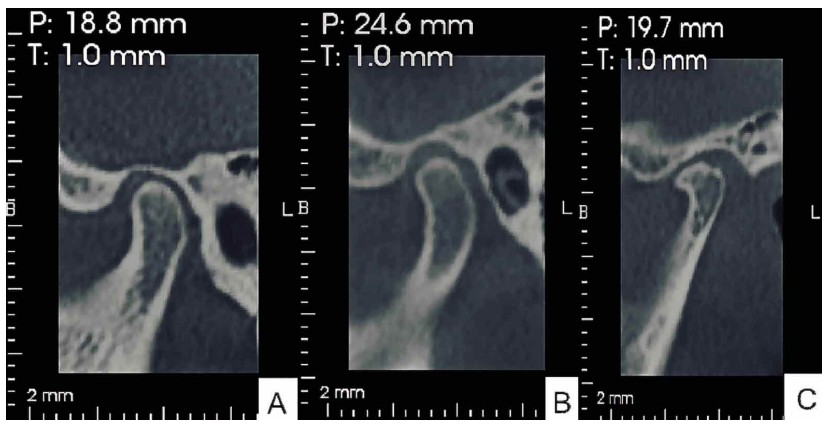

Fig. 2. Imagen de TCCB, corte sagital. A. Posición condilar posterior; B. Posición condilar central; C. Posición condilar anterior. 
Tabla I. Valores promedios, en milímetros, encontrados para los espacios articulares anterior, superior y posterior de cóndilos en posición central, posterior y anterior.

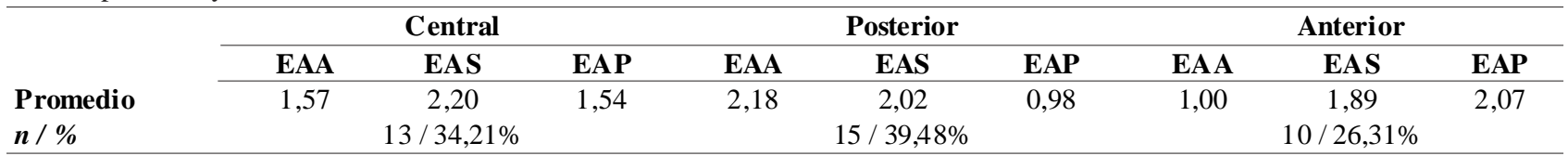

Los valores promedios de los espacios articulares obtenidos en cada grupo pueden ser apreciados en la Tabla I. No se observó diferencia estadística significativa entre los valores obtenidos para los lados derecho e izquierdo de la ATM.

\section{DISCUSIÓN}

En nuestro estudio la posición condilar posterior fue la más común en pacientes con TTM, sin embargo, las posiciones condilares anterior y central también fueron muy frecuentes, hecho que nos permite considerar que pacientes con TTM no presentan una posición condilar predominante, corroborando los hallazgos de Ren et al. (1995), BonillaAragon et al. (1999) y Pereira et al. Además, Bonilla-Aragon et al. afirman que la hipótesis de una ATM normal presentar necesariamente posición condilar céntrica no puede ser soportada. Por otra parte, observamos que un $65,79 \%$ de los cóndilos presentaron posición condilar no céntrica.

Algunos estudios señalan importantes diferencias entre los valores de los espacios articulares de articulaciones con y sin trastornos internos (Ren et al.; Kinniburgh et al.; Major et al., 2002; Gateno et al., 2004). Sin embargo, otros estudios han encontrado muy poca diferencia en estos valores comparando grupos sintomáticos y asintomáticos (Pereira \& Gavião). Bean \& Thomas (1987) encontraron poca diferencia en el estrechamiento del espacio articular entre pacientes sintomáticos y asintomáticos. Concluyeron que el $27 \%$ de los pacientes sintomáticos tenían disminución en el espacio articular anterior o posterior, mientras que el $30 \%$ de los pacientes asintomáticos mostraron una disminución de los espacios articulares anterior y posterior.

Ikeda \& Kawamura evaluaron los espacios articulares de ATMs sanas y encontraron para EAA: 1,3 mm; para EAS: 2,5 mm y para EAP: 2,1 mm. En nuestro estudio observamos que los valores promedios del EAP de cóndilos en posición central y posterior fueron menores que los valores reportados para individuos sanos. Es decir, en individuos con TTM los cóndilos ubicados en posición central y posterior presentan el EAP disminuido en comparación con individuos sanos. Observamos aun que el valor promedio del
EAS fue menor que lo reportado para individuos sanos con cóndilos en todas las posiciones (central, posterior y anterior), presentándose bastante disminuido en cóndilos con posición anterior. El EAA se presentó aumentado en cóndilos con posición central y posterior y disminuido en cóndilos con posición anterior.

Considerando nuestros hallazgos, concluimos que individuos con diagnóstico de TTM presentan las regiones anatómicas predisponientes a las alteraciones articulares con espacios articulares disminuidos cuando comparados con sujetos sin TTM. La posición posterior fue la más común en individuos con TTM, sin embargo, hubo gran variabilidad en la posición condilar lo que nos permite sugerir que una posición no céntrica del cóndilo no está necesariamente asociada con la presencia de TTM. Concluimos además que en individuos con TTM los cóndilos ubicados en posición central y posterior presentan EAP disminuido en comparación con individuos sanos.

ALVES, N.; DEANA, N. F.; SCHILLING, Q.A.; GONZÁLEZ, V. A.; SCHILLING, L. J. \& PASTENES, R. C. Assessment of TMJ condylar position and joint space in Chilean individuals with temporomandibular disorders. Int. J. Morphol., 32(1):32-35, 2014.

SUMMARY: The importance of the condyle-fossa relationship in the temporomandibular joint (TMJ) is not yet fully clear, however, some authors associate the non-central position of the head of the mandible in the mandibular fossa with temporomandibular disorder (TMD). Furthermore, other authors suggest that assessment of joint space is a diagnostic tool for assessing the TMJ alterations. The aim of this study was to evaluate the TMJ condylar position and joint space in Chilean individuals with TMD using cone-beam computed tomography. We selected 19 patients of "Unidad de Trastornos Cráneo Cervico Mandibulares (UCRACEM) - Universidad de Talca, Chile", who had a diagnosis of TMD according to the Research Diagnostic Criteria for Temporomandibular Disorders. The assessment of condylar position was performed using Pullinger \& Hollender technique and to evaluate the anterior joint space (AJS), superior joint space (SJS) and posterior joint space (PJS) was used Ikeda \& Kawamura technique. Posterior condylar position was found in $39.47 \%$ of condyles, central condylar position in $34.21 \%$ and anterior condylar position in $26.31 \%$. To condyles in central position we found: AJS $=1.57 \mathrm{~mm}$; SJS $=2.20 \mathrm{~mm}$; PJS $=$ $1.54 \mathrm{~mm}$. To condyles in posterior position we found: AJS = 
$2.18 \mathrm{~mm} ; \mathrm{SJS}=2.02 \mathrm{~mm} ; \mathrm{PJS}=0.98 \mathrm{~mm}$. To condyles in anterior position we found: AJS $=1.00 \mathrm{~mm}$; SJS $=1.89 \mathrm{~mm}$ and PJS $=2.07 \mathrm{~mm}$. We conclude that individuals diagnosed with TMD present anatomical regions predisposing to joint disorders with joint spaces diminished when compared with asymptomatic subjects. Furthermore, the posterior condylar position was the most common in individuals with TMD, however there was great variability in condylar position, therefore we suggest that a noncentralized position of the condyle is not necessarily associated with the presence of TMD. We further conclude that in individuals with TMD the condyles located in central and posterior position present EAP decreased compared with healthy individuals.

KEY WORDS: Condylar position; Joint space; Temporomandibular joint; Temporomandibular disorders.

\section{REFERENCIAS BIBLIOGRÁFICAS}

Alves, N. \& Cândido, P. L. Anatomia para o cirurgião-dentista. $3^{\text {a }}$ ed. São Paulo, Gen-Santos, 2013.

Bonilla-Aragon, H.; Tallents, R. H.; Katzberg, R. W.; Kyrkanides, S. \& Moss, M. E. Condyle position as a predictor of temporomandibular joint internal derangement. J. Prosthet. Dent., 82(2):205-8, 1999.

Bean, L. R. \& Thomas, C. A. Significance of condylar positions in patients with temporomandibular disorders. J. Am. Dent. Assoc., 114(1):76-7, 1987.

Dworkin, S. F. \& LeResche, L. Research diagnostic criteria for temporomandibular disorders: review, criteria, examinations and specifications, critique. J. Craniomandib. Disord., 6(4):301-55, 1992.

Gateno, J.; Anderson, P. B.; Xia, J. J.; Horng, J. C.; Teichgraeber, J. F. \& Liebschner, M. A. A comparative assessment of mandibular condylar position in patients with anterior disc displacement of the temporomandibular joint. J. Oral Maxillofac. Surg., 62(1):39-43, 2004.

Ikeda, K. \& Kawamura, A. Assessment of optimal condylar position with limited cone-beam computed tomography. Am. J. Orthod. Dentofacial Orthop., 135(4):495-501, 2009.

Katzberg, R. W.; Keith, D. A.; Ten Eick, W. R. \& Guralnick, W. C. Internal derangements of the temporomandibular joint: an assessment of condylar position in centric occlusion. $J$. Prosthet. Dent., 49(2):250-4, 1983.

Kinniburgh, R. D.; Major, P. W.; Nebbe, B.; West, K. \& Glover, K. E. Osseous morphology and spatial relationships of the temporomandibular joint: comparisons of normal and anterior disc positions. Angle Orthod., 70(1):70-80, 2000.
Major, P. W.; Kinniburgh, R. D.; Nebbe, B.; Prasad, N. G. \& Glover, K. E. Tomographic assessment of temporomandibular joint osseous articular surface contour and spatial relationships associated with disc displacement and disc length. Am. J. Orthod. Dentofacial Orthop., 121(2):152-61, 2002.

Matos, J. L. F.; Matos, M. F.; Ramos, F. M. M.; Almeida, S. M. \& Bóscolo, F. N. Avaliação do espaço articular em repouso de pacientes sintomáticos e assintomáticos de desordens temporomandibulares. Cienc. Odontol. Bras., 8(4):68-76, 2005.

Pereira, L. J. \& Gavião, M. B. Tomographic evaluation of TMJ in adolescents with temporomandibular disorders. Braz. Oral Res., 18(3):208-14, 2004.

Pereira, L. J.; Gavião, M. B.; Bonjardim, L. R. \& Castelo, P. M. Ultrasound and tomographic evaluation of temporomandibular joints in adolescents with and without signs and symptoms of temporomandibular disorders: a pilot study. Dentomaxillofac. Radiol., 36(7):402-8, 2007.

Pullinger, A. G.; Hollender, L.; Solberg, W. K. \& Petersson, A. A tomographic study of mandibular condyle position in an asymptomatic population. J. Prosth. Dent.,53(5):706-13, 1985.

Pullinger, A. \& Hollender, L. Variation in condyle-fossa relationships according to different methods of evaluation in tomograms. Oral Surg. Oral Med. Oral Pathol., 62(6):71927, 1986.

Ren, Y. F.; Isberg, A. \& Westesson, P. L. Condyle position in the temporomandibular joint. Comparison between asymptomatic volunteers with normal disk position and patients with disk displacement. Oral Surg. Oral Med. Oral Pathol. Oral Radiol. Endod., 80(1):101-7, 1995.

\author{
Dirección para Correspondencia: \\ Nilton Alves \\ Facultad de Odontología \\ Departamento de Odontología Integral Adulto \\ Universidad de La Frontera \\ Manuel Montt, 112 \\ Casilla 54-D \\ Temuco \\ CHILE
}

Email: niltonnalves@yahoo.com.br

Recibido: 21-09-2013

Aceptado: 19-11-2013 